\title{
A Study on the Effect of Inspection Time on Defect Detection in Visual Inspection
}

\author{
Ryosuke Nakajima $^{1, *}$, Keisuke Shida $^{2}$, and Toshiyuki Matsumoto ${ }^{1}$ \\ ${ }^{1}$ Aoyama Gakuin University, Kanagawa, Japan \\ d5613005@aoyama.jp, matsumoto@ise.aoyama.ac.jp \\ ${ }^{2}$ Nagaoka University of Technology, Niigata, Japan \\ shida@kjs.nagaokaut.ac.jp
}

\begin{abstract}
In order to consider the visual inspection utilizing the peripheral vision, this paper examines the inspection times that affect defect detection. The fixation duration and the distance between defect and the fixation point are experimental factors in determining the inspection time. As the result, in case of a large sized defect, the detection rate is high regardless of the fixation duration and the distance between the defect and the fixation point. In case of a small sized defect, when the fixation duration is longer and the distance between the defect and the fixation point is closer, the defect detection rate is higher. Moreover, as the result of conducting multiple linear regression analysis about the experiment factors, it is obtained that judging from standardized partial regression coefficient of factors, higher defect size, less the distance between defect and the fixation point, the higher fixation duration, is proved to improve the defect detection.
\end{abstract}

Keywords: Visual inspection, Inspection time, Peripheral vision, Effective field of view.

\section{Introduction}

In order to prevent the escape of defective products into markets, the strict inspection of products is carried out in Japanese manufacturing industries. There are two types of inspections, functional inspection and appearance inspection. In functional inspection, the motion and efficiency of the products is inspected and in appearance inspection, small visual defects like scratch and stain are checked. Automation of functional inspection has advanced whereas the automation of appearance inspection is very difficult and greatly depends on the visual inspection by workers' eyes. Generally, the area which can be processed by the visual stimulus is called field of vision [1]. In the field of vision, the area within $1 \sim 2\left[^{\circ}\right]$ of the center of the retina is called central visual field and the surrounding area is called peripheral vision. The spatial resolution of the field of vision reduces remarkably when moving far from the center of retina [2]. This is due to the effect of two types of cells in that are distributed in the field of vision. Two different cells distributed un a field of vision have an influence on the human sight, one is a cone cell having the functions to distinguish colors and to view objects clearly in the central visual field. The other is a rod cell having the functions to 
distinguish luminance sensitively and cannot distinguish colors in the peripheral visual [3].

This difference in the distribution of the cells affects the human behavior of searching for something. When humans search for objects, first a wide range is searched using peripheral vision and then the item is searched using the central vision which has high precision. To increase the efficiency of search by reducing the information the eye needs to process. Specifically, low level processes like clustering and detection of properties use peripheral vision and then high level processes like discrimination of object is carried out [4].

However, in actual visual inspection, in order to thoroughly inspect, the central vision is mainly used. This increases the amount of information that needs to be processed and is thought to reduce the work efficiency. In other words, there is the need to use both central and peripheral visions properly and therefore the development of the new visual inspection method that does not only use the central vision, but also the peripheral vision is needed [5]-[9].

In actual visual inspection, the detection of scratches, the stain irregularity in color etc. is necessary and the deciding whether the product is defective or non-defective depends on the size and depth. Furthermore, high-mix, low-volume production is advanced recently and the inspection of various products is necessary which causes other difficulties, for instance, same size scratches can be within the standard for one object but otherwise for another object. Furthermore, the standard is different according to the need of the customer, even though is for the same object. If the defect that is within the standard is decided as the defective product, over kill occurs which causes financial loss, and if the defect that is not within the standard is decided as nondefective, Escape occurs which causes the flow of defective product to markets.

Actually, inspection methods should be changed according to the quality demand of the customer, but in actual visual inspection, single inspection method is continuously used since there is the limit in the inspection time in order to maintain fixed productivities. Therefore, in this study, appropriate inspection method is examined by considering the factors that affects the inspection time.

\section{Factors of Affecting the Inspection Time}

In visual inspection, the time required to inspect products can be defined using the number of fixation points and the time needed for fixing the fixation points. Normally, the characteristics in the design of the product are used as fixation points, and by fixing these points in order assure that the product is thoroughly inspected. The inspection time increases when the fixation duration of the fixation points and the number of fixation points are increased.

The length of the inspection time depends on the effective visual field range of the peripheral vision and the fact that the effective visual field range widens when the fixation duration increases has been shown [10]. When the effective visual field range widens, the range of inspection in one fixation widens and an increase in the detection rate can be expected. Moreover, when the effective visual field range increases, overlapping area of field of vision also increases and the increase in the detection rate can be expected since the same area is viewed number of times. 
Additionally, when the number of fixation points is increased, the probability that the fixation point is placed near the defect increases. Since the distance between the fixation point and the defect is less, the judgment of the defect becomes possible because it is in the field of vision with high spatial resolution and increase in the detection rate can be expected [11].

In this way, both the fixation duration and the number of fixation points can be considered as factors that affect the defect detection, but the relationship between the fixation duration and the number of fixation point has not been clarified. Therefore, when the manager of the production line changes the inspection time, it is difficult whether to increase the fixation duration or to increase the number of fixation points.

Thus, in this study, the effect of fixation duration and the number of fixation points on the defect detection rate for various standards are examined.

\section{Experimental Method}

\subsection{Experimental Task}

As the experimental task, the target object which is the black circle of $1 \mathrm{~cm}$ in an area of A4 size as shown in Fig. 1 is displayed on the monitor (FlexScan SX2462W). By considering the fact that the field of vision of human is horizontally wide [12], the target object is also made horizontally wide. Furthermore, in order to lead inspection by the peripheral vision, the fixation point is placed in the center of the target object and the subject is made to fixation only at the fixation point. In order to make the viewing angle of subjects the same, the stand is placed at $40 \mathrm{~cm}$ from the monitor where the lower chin of the subject is positioned. The experiment layout is shown in Fig. 2. Furthermore, the brightness of the monitor is $199[\mathrm{~cd} / \mathrm{m} 2]$ and the lighting density calculated from the position of keyboard is $810[1 \mathrm{x}]$.

Subjects fixation at the fixation point and inspect the whole target object by the peripheral vision for five different fixation durations, from $0.25 \mathrm{sec}$ to $1.25 \mathrm{sec}$, which are decided by the experimenter. If there is not a defect, the subject presses the Space key and the next target object is displayed. If the defect is detected, the subject presses enter key. Furthermore, in order to verify that the subject is inspecting the target using the peripheral vision, the eye movement is monitored using an eye tracker (NAC EMR-9) which is fixed on the subject during the experiment.

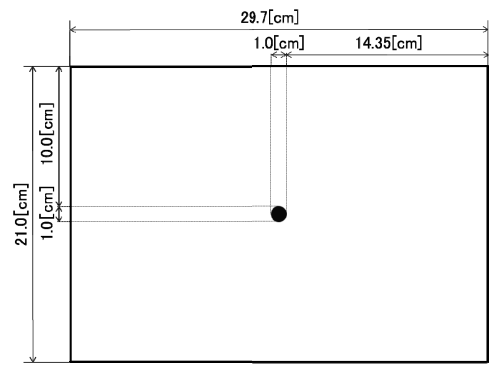

Fig. 1. Target object

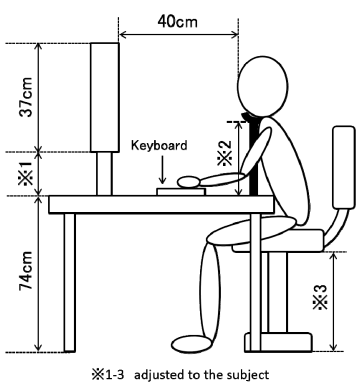

Fig. 2. Experimental layout 


\subsection{Experimental Factors}

\subsubsection{Fixation Duration}

In order to control the fixation duration of the subject, the display time of the target object is limited. Five sets of display times are used in this experiment, which are $0.25 \mathrm{sec}, 0.50 \mathrm{sec}, 0.75 \mathrm{sec}, 1.00 \mathrm{sec}$ and $1.25 \mathrm{sec}$. The reason for fixing the display time of the target work is that, during preliminary experiment, the detection rate for the display time of $0.25 \mathrm{sec}$ was $50 \%$. Therefore, if the display time is less than $0.25 \mathrm{sec}$, subjects will not be able to find most of the defects.

\subsubsection{Distance between the Defect and the Fixation Point}

The target object is divided into thirty six parts (horizontally $6 \times$ vertically 6 ) as shown in Fig.3 and the defect is placed in the center of either one of these parts. As in Fig. 3, the parts are divided in to nine types from a to $i$, according to the distance from the fixation point. The distance from the fixation point to the center of each part are a: $3.0 \mathrm{~cm}$, b: $5.8 \mathrm{~cm}, \mathrm{c}: 7.6 \mathrm{~cm}, \mathrm{~d}: 9.1 \mathrm{~cm}$, e: $11.4 \mathrm{~cm}, \mathrm{f}: 12.5 \mathrm{~cm}, \mathrm{~g}: 13.4 \mathrm{~cm}$ and i: $15.1 \mathrm{~cm}$.

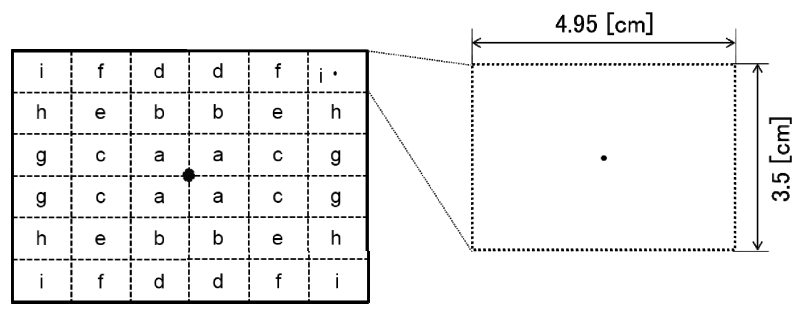

Fig. 3. Target object

\subsubsection{Size of the Defect}

The sizes of the defects are of four types with diameters of $0.06 \mathrm{~cm}, 0.08 \mathrm{~cm}, 0.10 \mathrm{~cm}$, $0.12 \mathrm{~cm}$. There are defects of various types in manufacturing industries, for instance, in painting processes, there are point shaped defects like iron powder and line shaped defects caused by threads. However, when the size of the defects become small, the shape of the defect need not be considered and all the defects can be summarized as a circle (point). Specially, in this experiment where small defects that are difficult to detect are targeted, the shape of the defect is fixed as a circle. The background color is set as white. The four types of defects are placed in 36 parts, which makes the total number of the target object as 144 ( 36 parts $\times 4$ types).

\subsection{Experimental Procedure}

Fifteen subjects (ten male, five female) from 22 to 24 years of age are used in this experiment. In order to make the subjects get use to the experiment, the procedure of the experiment is explained and they are made to do a preliminary experiment and subjects with corrected eyesight of more than 1.0 are used. Additionally, subjects those who use glasses or contact lens are not used in this experiment, so that there is 
no effect on the eye camera readings. The proportion of the defect in the experiment is set to $20 \%$ and 720 target objects are inspected. This is repeated for the each fixation duration. The reason for setting the defect proportion as $20 \%$ is that it was found during the preliminary experiments that there is a tendency of the subjects to use the central vision when the defect proportion increases.

The experiments for the five types of fixation duration were carried out in two days by considering the fatigue of the subjects. The room temperature is set between 18 and $22^{\circ} \mathrm{C}$ and humidity between 40 and $50 \%$.

\section{$4 \quad$ Experimental Results}

\subsection{Individual Characteristics of Subject}

Using the defect detection rate, the effect of fixation duration and the distance between the fixation point and the defect is examined, but there is the possibility that the individuality of the subject will affect the result. As the result of this experiment, the uniformity of results for various subjects was verified. The defect detection rate is expressed in Eq. (1).

$$
\text { the defect detection rate }[\%]=\frac{\text { the number of detected defects }}{\text { the number of total defects }} \times 100
$$

The defect detection rate of the each subject according to the fixation duration is shown in Fig. 4, from this result it is observed that when the fixation duration increases, the defect detection rate increases for all the subjects, but this tendency is not seen for some of the workers. There were the subjects who had high defect detection rate even if the fixation duration is short. Even if there were differences between the subjects, there is no outlier value for the defect detection rate. Moreover, there is no outlier value in the case of distance between the fixation point and the defect and the size of the defect, therefore, the data of the fifteen subjects were used and examined.

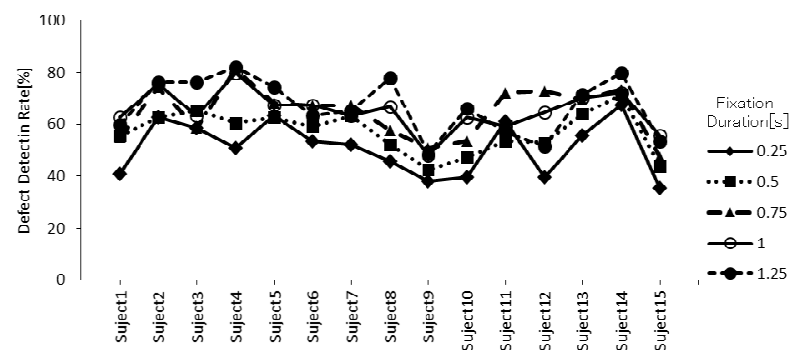

Fig. 4. Defect detection rate of the each subject according to the fixation duration 


\subsection{Effect of the Fixation Duration and the Distance between the Defect and the Fixation Point on the Defect Detection Rate}

The effect of the fixation duration and the distance between the defect and the fixation point on the defect detection rate is shown in Fig. 5a and b, and the effect of the size of the defect on the defect detection is shown in Fig. 5c. The analysis of variance of three-way layout with the fixation duration of the defect (5), the distance between the defect and the fixation point (9) and the size of the defect (4) as factors is executed. As the result, a significant difference of $1 \%$ is observed for the main effect of the fixation duration, the distance between the defect and the fixation point and the size of defect. Moreover, the significant difference of $1 \%$ is observed for the mutual interaction in which the mutual interaction of the distance between the defect and the fixation point is excluded. The reason for this can be seen when this result is divided according the size of the defect, as shown in Fig. 6a, it can be seen that the effect of the fixation duration and the distance between the defect and the fixation point on the defect detection rate is less when the size of the defect is large. On the other hand, there is the relation between the fixation duration and the distance between the defect and the fixation point as can be shown in Fig. 7. It is confirmed that the defect detection rate is increased when the fixation duration is long and the distance between the defect and the fixation point is short.

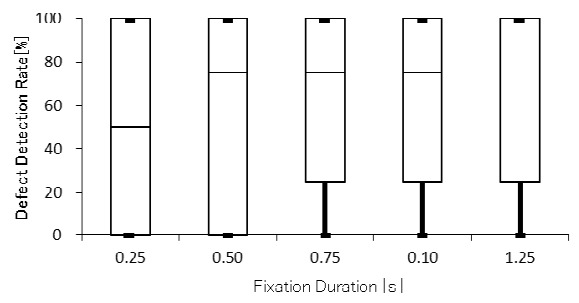

a. Defect detection rate of each fixation duration

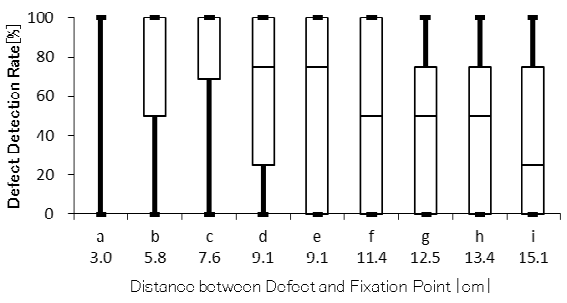

b. Defect detection rate of each distance between the defect and the fixation point

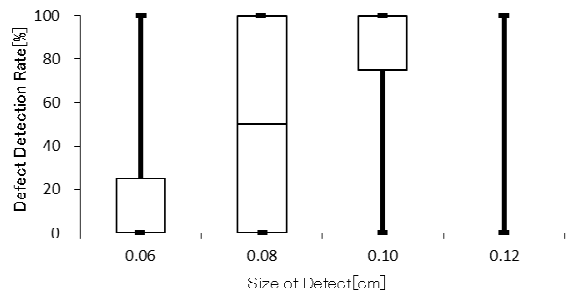

c. Defect detection rate of each size of defect

Fig. 5. Distribution of defect detection rate 


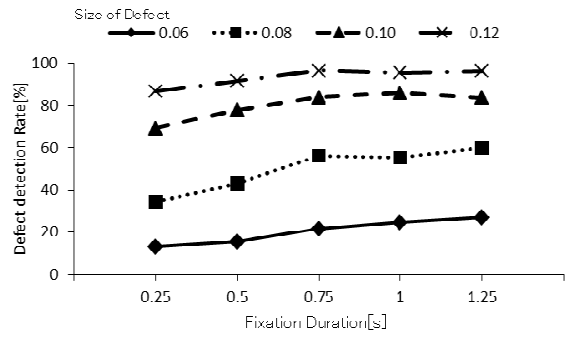

a. Fixation duration and size of defect

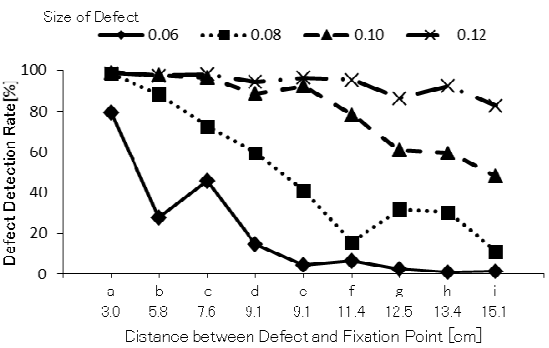

b. Distance between fixation point and defect and size of defect

Fig. 6. Interaction between the two factors

Table 1. Analysis of variance

\begin{tabular}{|c|c|c|c|c|c|}
\hline Variation factor & Sum of squares & Flexibility & Mean square & F-number & Decision \\
\hline $\begin{array}{c}\text { Fixation duration of } \\
\operatorname{defect}(\mathrm{A})\end{array}$ & 99688.43 & 4 & 24922.11 & 65.49 & $* *$ \\
\hline $\begin{array}{l}\text { Distance between defect } \\
\text { and fixation point(B) }\end{array}$ & 876307.41 & 8 & 109538.43 & 287.84 & $* *$ \\
\hline Size of the defect (C) & 2152230.56 & 3 & 717410.19 & 1885.17 & $* *$ \\
\hline$A \times B$ & 15090.74 & 32 & 471.59 & 1.24 & \\
\hline $\mathrm{A} \times \mathrm{C}$ & 16572.69 & 12 & 1381.06 & 3.63 & $* *$ \\
\hline $\mathrm{B} \times \mathrm{C}$ & 382677.78 & 24 & 15944.91 & 41.90 & $* *$ \\
\hline $\mathrm{A} \times \mathrm{B} \times \mathrm{C}$ & 56581.48 & 96 & 589.39 & 1.55 & ** \\
\hline Error & 959000.00 & 2520 & 380.56 & & \\
\hline Total & 4558149.07 & 2699 & & & \\
\hline
\end{tabular}

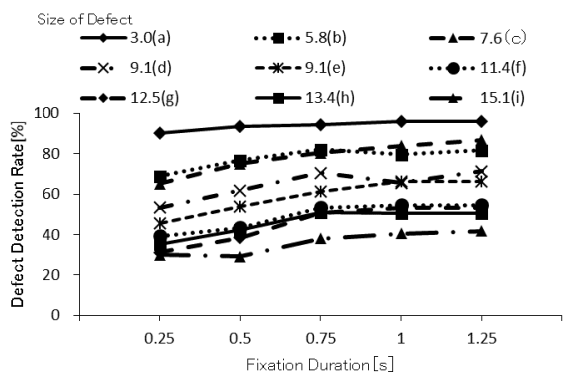

Fig. 7. Relation between the fixation duration and the distance between the defect and the fixation point 


\section{Discussion}

\subsection{Inspection Method Corresponding to the Requirements Regarding the Defects}

In order to examine the level of the effect of the fixation duration, the distance between the defect and the fixation point and the size of the defect on the defect detection rate, a multiple regression analysis is carried out with these three factors as decision variable and the defect detection rate as the objective variable. As the result, the standard partial regression coefficient of the decision variables is as shown in Table 2, where the coefficient of size of the defect is found to be the biggest. In short, it is seen that the main factor that effect the defect detection rate in the visual inspection is the size of the defect and it reduces in the order of the distance between the fixation point and the defect, and the fixation duration.

This result also supports the fact that there is a significant difference in the mutual interactions including the size of the defect. That is, when the size of the defect is big, the defect detection rate is high and does not depend on the fixation duration or the distance between the defect and the fixation point as shown in Fig. 6. This can be also confirmed through use of the value of the standard regression coefficient of the size of the defect which is 0.7 , a high value. On the other hand, for small defects i.e. when the demanding quality standards are high, the fixation duration and the distance between the defect and the fixation point should also be considered.

However, when the fixation duration and the distance between the defect and the fixation point are compared, it is shown that reducing the distance between the defect and the fixation point affects the defect detection rate more than increasing the fixation duration. In other words, if there is the necessity to find small defect and the quality demand of the customer is high, the first measure should be to increase the number of fixation points.

Table 2. Result of multiple regression analysis

\begin{tabular}{|c|c|c|c|}
\hline Independent variable & $\begin{array}{c}\text { Partial regression } \\
\text { coefficient }\end{array}$ & $\begin{array}{c}\text { Standard partial } \\
\text { regression coefficient }\end{array}$ & $\begin{array}{c}\text { Significant } \\
\text { difference }(* * 1 \%)\end{array}$ \\
\hline Size of defect & 1248.9 & 0.76 & $* *$ \\
\hline Fixation duration & 16.2 & 0.16 & $* *$ \\
\hline $\begin{array}{c}\text { Distance between defect } \\
\text { and fixation point }\end{array}$ & -4.8 & -0.48 & $* *$ \\
\hline
\end{tabular}

Decision variable $=0.84$ Adjusted $\mathrm{R}^{2}=0.83$.

\subsection{Effect of the Position of the Fixation Point and the Defect on the Defect Detection Rate}

The effective field of vision of this experiment is examined. The area that has high defect detection rate is painted with dark color and that with low defect detection rate is colored with light color for each fixation duration. Ten colors are used for each $10 \%$ and the result is shown from Fig. 8a to e. As the result, for all fixation duration, 
the effective field of vision is horizontally wide and the area increases when the fixation duration increases. Generally, the field of vision of humans is horizontally wide [13] and the information inside a horizontal angle of vision of $30^{\circ}$ and the vertical angle of vision of $20^{\circ}$ can be effectively processed [14]. The angle of vision in this experiment is $21.2^{\circ}$ horizontally and $14.8^{\circ}$ vertically, which is clearly inside the above said range, but it can be clearly seen that even inside this range of the angle of the vision, the defect detection rate is not uniform.

Furthermore, the shape of the effective field of vision in vertical direction is examined. Corresponding from Fig 8a to e, parts 9 and 10 and parts 27 and 28 were taken for analysis. As the result, instead of the distance of these parts from the fixation point being same, for all the fixation durations there is a difference of an average of $10 \%$ in the detection rate and anisotropy is seen for the characteristics of the field of the vision in the vertical direction. In other words, the distance between the defect and the fixation point does not affect the detection rate, but there is difference in the detection rate according to the distance between the fixation point and the defect in the horizontal position and the vertical position. This means that it is clear that the position of the defect with regard to the fixation point also causes effect on the detection rate. That is, when fixing the fixation point, it should be considered that not only the distance but also the position in the field of vision causes effect on the defect detection rate.

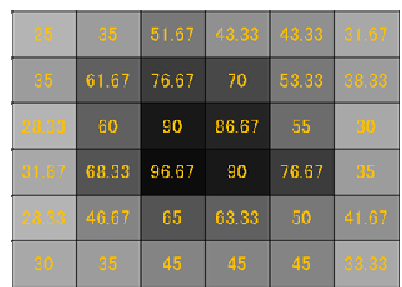

a. 0.25

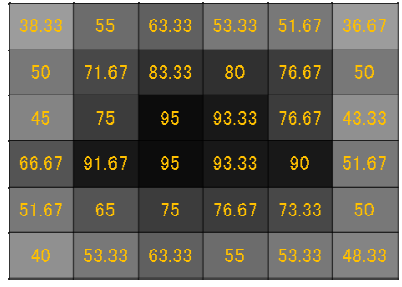

d. 1.00

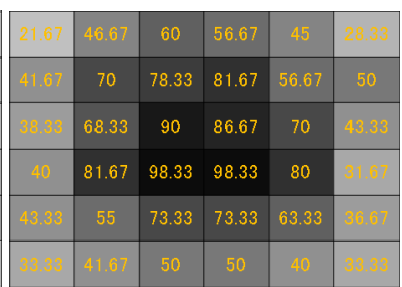

b. 0.50

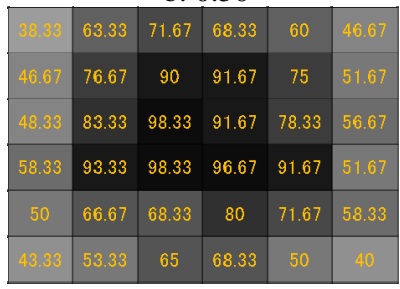

e. 1.25

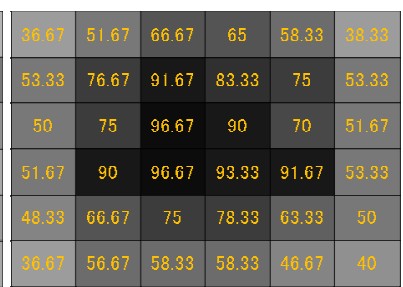

c. 0.75

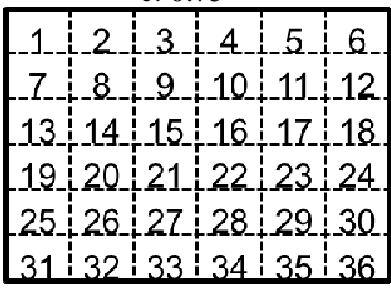

f. number of parts

Fig. 8. Relation between the fixation duration and the distance between defect and fixation point

\section{Conclusion}

Visual inspection is a sensory inspection, in which there is difference in result according to the individuality and the skill of the worker. This has been a major problem in the manufacturing industries where the standardization of the work is 
required. In addition, there have been various measures like evaluation and training of workers in order to increase the inspection technique, but there have not been any considerable results. Therefore, in this study, the potentiality of human sight mechanism using the peripheral vision was examined, for that, the effect of fixation duration, distance between the defect and the fixation point and the size of the defect on the defect detection rate was examined.

As the result, the effect of the size of the defect was found to be largest and it was shown that in order to find large defects, the number of fixation points could be reduced and the inspection time also would be reduced. On the other hand, while inspecting for small defects, the defect detection rate could be increased by increasing the number of fixation points and reducing the distance between fixation point and the defect which increased as the inspection time. Additionally, it was discovered that while placing a fixation point on the targeted object, not only the distance between the defect and the fixation point had to be considered, but also the position of the defect.

In future studies, the characteristics of the effective field of vision in visual inspection should be examined and also, using the result of this study, the effect of increasing the fixation points and increasing the fixation duration should be tested in actual working lines in the manufacturing industries.

Acknowledgement. This study was supported by JSPS Grant-in-Aid for Young Scientists (A) Grant Number (23681034).

\section{References}

1. The Japan Society of applied Physics: Seiri Kougaku, pp. 1-302. Asakura Publishing (1975)

2. Ikeda, M.: Meha Naniwo Miteiruka, pp. 1-289. Heibonsya (2004)

3. Ujiie, Y., Saita, S.: Vision, Handbook of human behavior measurement, p. 165. Asakura Publishing (2003)

4. Yoshida, C., Toyoda, M., Sato, Y.: Vision System Model with Differentiated Visual Fields. Information Processing Society of Japan 33(8), 1032-1040 (1992)

5. Sasaki, A.: Syuhenshi Mokushikensahou (1). The Japan Institute of Industrial Engineering Review 46(4), 65-75 (2005)

6. Sasaki, A.: Syuhenshi Mokushikensahou (2). The Japan Institute of Industrial Engineering Review 46(5), 61-68 (2005)

7. Sasaki, A.: Syuhenshi Mokushikensahou (3). The Japan Institute of Industrial Engineering Review 47(1), 55-60 (2006)

8. Sasaki, A.: Syuhenshi Mokushikensahou (4). The Japan Institute of Industrial Engineering Review 47(2), 53-58 (2006)

9. Sasaki, A.: Syuhenshi Mokushikensahou (5). The Japan Institute of Industrial Engineering Review 47(3), 67-72 (2006)

10. Kumada, T., Kuchinomachi, Y., Saida, S.: The Properties of function Visual Fields on Visual Search: Evidence from Manual Reaction Time and Saccadic Eye movement. The Japanese Journal of Psychonomic Science 14(2), 75-85 (1996)

11. Jacobs, R.J.: Visual resolution and Contour Interaction in the Fovea and Periphery. Vision Research 19(11), 1187-1195 (1979) 
12. Chaikin, J.D., Corbin, H.H., Volkmann, J.: Mapping a field of short-time visual search. Science 138, 327-328 (1962)

13. Yamanaka, K., Nakayasu, H., Miyoshi, T., Maeda, K.: A Study of Evaluating Useful Field of View at Visual Recognition Task. The Japan Society of Mechanical Engineers 72(719), 2248-2256 (2006)

14. Hatakeda, T.: Handbook of human behavior measurement, p. 585. Asakura Publishing (2003) 